\title{
PROGRAMAS DE EDUCACIÓN SEXUAL EN PANAMÁ ${ }^{1}$
}

\section{Claude Vergès*}

Resumen: ¿Cuál es el lugar del placer en los programas de educación sexual? En Panamá, como en otros países de América Latina, la violencia contra niños y niñas y contra las mujeres no les permite reconocer la propiedad de su propio cuerpo y menos su derecho al placer. Los programas actuales sobre educación sexual, prevención del embarazo y SIDA promueven el uso del preservativo y la abstinencia pero no hablan de la ética del placer. Frecuentemente, el personal sanitario y educativo no está preparado para hablar sobre el tema. El uso del placer sexual como mercancía en los medios de comunicación introduce mayor confusión. La bioética debe integrar los estudios de la psicología, la antropología y un sentido de humanidad que permitan a este personal trabajar con las personas hacia la apropiación de su integridad como ser humano.

Palabras clave: placer, educación sexual, salud, derechos humanos

\section{PROGRAMS ON SEXUAL EDUCATION IN PANAMA}

\begin{abstract}
Which place occupies pleasure in sexual education programs? In Panama, as in other Latin American countries, violence against children and women does not allow people to realize own bodylines and less their right to pleasure. Present programs about sexual education, pregnancy prevention and AIDS promote the use of preservatives and abstinence but do not include the ethics of pleasure. Frequently, the health and training personnel are not prepared to speak about the topic. The use of sexual pleasure as a merchandise in media creates greater confusion. Bioethics must integrate psychological and anthropological studies and a sense of humanity to allow this personnel to work with persons towards the integral appropriation of human beings.
\end{abstract}

Key words: pleasure, sexual education, health, human rights

\section{PROGRAMAS DE EDUCAÇÃO SEXUAL NO PANAMÁ}

Resumo: Qual é o lugar do prazer nos programas de educação sexual? No Panamá, como em outros países da América Latina, a violência contra meninos e meninas e contra as mulheres não lhes permite reconhecer a propriedade dos seus corpos e muito menos os seus direitos ao prazer. Os atuais programas sobre educação sexual, contracepção e AIDS promovem o uso do preservativo e a abstinência, mas não se referem à ética do prazer. Frequentemente, os profissionais da saúde e da educação não se encontram preparados para tratar sobre o tema. O uso do prazer sexual como mercadoria nos meios de comunicação acende ainda mais o conflito. A bioética deve integrar os estudos da psicologia, da antropologia e o sentido de humanidade de modo a permitir que tais profissionais possam trabalhar com as pessoas a apropriação de sua integridade como ser humano.

Palavras chave: prazer, educação sexual, saúde, direitos humanos

1 Trabajo presentado en el IX Congreso Internacional de Bioética, Sydney-Australia, 2004, corregido en marzo de 2007.

* Profesora de Ética Médica y Bioética, Facultad de Medicina, Universidad de Panamá, Panamá

Correspondencia: cverges2004@yahoo.es 


\section{Introducción}

El placer es una de las sensaciones más importantes del ser humano para la determinación de su conducta cotidiana y, particularmente, para su vida sexual. Sin embargo, los programas actuales de educación sexual, control del embarazo y prevención del SIDA promueven el uso del preservativo y la abstinencia sexual pero no hablan del placer. Según la moral cristiana, el placer es vergonzoso y, frecuentemente, el personal de salud y -más aún-el educativo no están preparados para hablar del tema. La promoción del placer sexual sin límites en los medios de comunicación agrega más confusión.

El desarrollo de la sensación de placer se inicia desde el nacimiento, influenciado por factores fisiológicos, psicológicos y culturales. Los fisiológicos están determinados genéticamente y por cambios hormonales; los factores psicológicos pertenecen a cada individuo y dependen de las relaciones familiares que permitirán al niño o a la niña definirse como persona.

En América Latina, la violencia contra niñas y niños y contra las mujeres no les permite reconocer a su cuerpo como propio, ni su derecho al placer. Sin embargo, se observan adelantos con la inclusión de programas contra la violencia desarrollados por los ministerios de salud, a partir de las conferencias internacionales de las mujeres en México y en Beijing, y del reconocimiento de la violencia como un problema ético de Derechos Humanos.

Para las sociedades indígenas de Panamá la sexualidad en sí no es un problema ético sino una parte del ser humano, y el placer sexual es un derecho de la edad adulta al cual acceden los jóvenes hombres y mujeres cuando tienen derecho de participar en las decisiones de la comunidad ("comer en el plato de los adultos") 2 .

2 Expresión de un dirigente Kuna al referirse a la ceremonia de la pubertad y al derecho de participación social.
¿Qué lugar tiene el concepto de placer en los programas de educación sexual? ¿Cómo, cuándo y dónde dar esta educación? ¿Qué piensa la gente de esta visión? ¿Qué puede aportar la bioética a estos programas?

\section{Reflexión}

En 2003 participamos en un programa de educación en salud sexual y reproductiva en el marco de un curso sobre derechos humanos desarrollado por una organización no gubernamental. Al hablar de placer en las diferentes etapas de la vida y de la necesidad social del control de estos placeres, los participantes exhibieron una primera reacción de confusión y extrañeza, para luego participar de manera muy entusiasta y presentar alternativas de formación de la autoestima y del control de sí mismo. Además, expresaron que, tanto en los programas del Ministerio de Salud como en los programas escolares sobre salud sexual y reproductiva, nunca se había hablado del placer y que era un tema necesario para una visión integral. Encontramos la misma reacción en los estudiantes de medicina durante el curso de Ética Médica y Bioética. Al revisar los contenidos de los programas oficiales de educación sexual del Ministerio de Educación y del Ministerio de Salud en Panamá, comprobamos que no se mencionaba el tema; una nueva revisión en 2005 fue también negativa. Sin embargo, observaciones de nuestra experiencia de trabajo sobre los temas de salud sexual y reproductiva, equidad de género y derechos humanos con diferentes grupos $^{3}$ revelan que hay deseos de entender la relación entre placer y felicidad y de no ver siempre a la sexualidad como negativa.

3 Los cuales siempre fueron advertidos y estuvieron de acuerdo en que las conversaciones podrían ser utilizadas como referencias, preservando siempre la confidencialidad de las fuentes (anonimato) y la privacidad de los datos (exclusión de datos personales). 


\section{Derechos sexuales y reproductivos}

Panamá tiene 3,2 millones de habitantes, de los cuales 1,25 millones son menores de 19 años: un tercio entre 10 y 14 años y un poco menos del tercio de 15 a 18 años(1). En 2003, la tasa de embarazos en adolescentes era de $18,4 \%$, y $11,6 \%$ de los menores de 18 años tenían SIDA(1). Estas tasas son diez veces más altas que en los países desarrollados; un estudio del Allan Gutmatcher Institute comparó el embarazo en adolescentes de Francia, Suecia, el Reino Unido, Canadá y Estados Unidos; la tasa de embarazo en el grupo de 15 a 19 años en Francia y Suecia era de 20 y 25 por 1.000 mujeres, respectivamente, y cuatro veces más alto en Estados Unidos (84 por 1.000)(2). Cada año, cerca de medio millón de mujeres fallece por causas relacionadas con el embarazo y el parto en el mundo. El riesgo de morir de las mujeres jóvenes durante estos procesos es dos veces más alto que en las mujeres mayores de 20 años, y éste aumenta de cuatro a cinco veces en jóvenes menores de 15 años. El embarazo es la causa principal de muerte de las mujeres entre 15 y 19 años en el mundo(2).

Por otra parte, la mitad de todas las nuevas infecciones por HIV en el mundo ocurre en jóvenes de 15 a 24 años, y la mayoría son niñas. La Organización Mundial de la Salud (OMS) estima que cerca de 330 millones de nuevas infecciones de transmisión sexual ocurren cada año, y que un tercio de los casos se produce en jóvenes menores de 25 años; $62 \%$ de todos los jóvenes que vivían con HIV/SIDA en 2001 eran mujeres(3). En Panamá, el aumento de la tasa de SIDA es más rápido en las mujeres mayores de 20 años que en los hombres.

En el documento de acciones de la Conferencia Internacional sobre Población y Desarrollo $(\mathrm{ICPD}+5)$, la comunidad internacional aprobó una definición de salud reproductiva que incluye su reconocimiento como un derecho humano, tal como aparecía en los acuerdos internacionales de derechos humanos. El programa de acción admite el derecho de las personas y de las parejas a elegir si quieren hijos, cómo y con qué frecuencia, y subraya el derecho de las personas de acceder a "servicios de salud reproductiva", incluyendo los métodos de regulación de la fertilidad "cuando no están contra la legislación local". Estos derechos sexuales incluyen los derechos de mujeres, jóvenes y homosexuales y lesbianas a tomar sus propias decisiones en cuanto a su sexualidad(4). Desde el inicio, los países latinoamericanos - con una mayoría católica conservadora-, los países musulmanes y el Vaticano expresaron sus reservas(5).

A pesar de ello, varios países latinoamericanos, como Panamá, desarrollaron un discurso abierto sobre los temas de salud sexual y reproductiva y derechos humanos entre 1995 y 2002. Sin embargo, las iglesias norteamericanas conservadoras lideraron una campaña de oposición al aborto, la planificación familiar, la equidad de género y las personas no casadas que iniciaron al interior de Estados Unidos, pero que se extendió rápidamente a los países latinoamericanos. Por su parte, la jerarquía católica siempre se opuso al uso de los preservativos y a la educación sexual, y prefiere hablar de la abstinencia como "sexo responsable"(6). En Panamá, esta campaña ha sido apoyada por los teólogos bioeticistas y dirigida principalmente a convencer a médicos y educadores; los obstetras han sido la especialidad de particular atención por parte de estos grupos.

En la reforma al Código Penal propuesta en diciembre de 2006 las iglesias conservadoras lograron introducir la figura de "la objeción de conciencia” por parte de los médicos en caso de aborto 4 . El término "embarazo seguro" ha sido

4 Este derecho no ha sido reglamentado y puede poner en peligro a la vida de las mujeres en los lugares donde hay un solo médico y pobre capacidad de referencia a otro servicio de salud. 
secuestrado por estos grupos para introducir el concepto de "vida dependiente" desde la concepción, olvidándose de las tasas de mortalidad materna. En este marco, es importante clarificar conceptos y salvaguardar los derechos de los grupos vulnerables a la salud sexual y reproductiva: el género, la edad, la clase socioeconómica y la pobreza, la educación y la violencia son factores importantes de restricción de la libertad de decisión(7) a los cuales no se pueden agregar otros.

Estos grupos conservadores se oponen también al concepto de "servicios de salud reproductiva", argumentando que pueden abrir la puerta al aborto, y prefieren el término de "cuidados" para el acceso a los tratamientos médicos(6). Algunos consideran que esta visión dicotómica es similar a las propuestas neoliberales de separar los servicios de atención a las enfermedades y la promoción de la salud. Pero no debemos olvidar la definición de la salud de la OMS que recoge la integralidad del cuerpo humano en su contexto social, psicológico y cultural, lo que implica que los servicios de salud respondan a los derechos de las personas al control sobre la sexualidad y la fertilidad propia y no estén restringidos para el tratamiento de las enfermedades(8). Sin embargo, los programas de educación sexual en las escuelas públicas se limitan a clases de anatomía sexual y de desarrollo del embrión, además muy superficiales. En los servicios de salud, estos programas han integrado los conceptos de equidad de género, autoestima y rechazo de la violencia, pero su desarrollo depende del grado de compromiso del personal de salud. Aunque los servicios públicos de salud ofrecen todavía métodos contraceptivos de bajo costo o gratuitos, el abastecimiento de estos insumos es irregular, lo que disminuye su utilización.

La defensa del reconocimiento de las tradiciones culturales, promovida por los grupos conservadores, se limita a las tradiciones es- pañolas del siglo XVIII y rechaza los valores tradicionales de los grupos indígenas respecto de la ley natural como ciclo de reproducción y de muerte, y la participación de la mujer en este proceso con pleno derecho (independientemente de la estructura patriarcal de estos grupos). En las discusiones con los grupos focales se evidenció que las mujeres panameñas utilizan métodos anticonceptivos de manera similar a la evolución de los demás países en desarrollo(9), tal como lo reconoce la Plataforma de Acción de Beijing. El uso del preservativo está menos difundido, porque debemos tomar en cuenta los prejuicios machistas sobre la "pérdida de potencia sexual" con su uso.

Contra la voluntad de los adolescentes, y de la opinión de una amplia mayoría de participantes de los grupos con los cuales hemos trabajado, los derechos parentales son utilizados por grupos conservadores para prevenir el acceso de las adolescentes a los anticonceptivos y contrarrestar la información y la orientación sobre salud sexual de los programas de salud. Estos grupos argumentan que los padres tienen el poder de decisión sobre la salud sexual y reproductiva de sus hijos e hijas. Sin embargo, el desarrollo de los derechos de la niñez y de la adolescencia ha favorecido el reconocimiento de la necesidad de contar con su asentimiento o consentimiento voluntario para participar en investigaciones y en las decisiones sobre su salud (12-14 años). Si los Estados reconocen que los niños pueden trabajar desde los 14 años para ayudar a la economía familiar, ¿no es contradictorio negarles su derecho a decidir sobre su futuro y gozar de una vida plena? La promoción y la protección de los derechos de niños, niñas y adolescentes a la educación no pueden ser negadas. La visión "apocalíptica” de los adultos, incluyendo personal de salud y educadores, sobre las enfermedades de transmisión sexual y las orientaciones sexuales diferentes a la heterosexualidad asusta a los jóvenes y difi- 
culta su participación, por lo que los programas no cumplen con su cometido.

\section{El placer y la sexualidad}

Para las poblaciones indígenas de Panamá (y los Kunas han sistematizado este pensamiento), el placer es una necesidad natural y, por lo tanto, está limitado por las necesidades del individuo y/o del grupo. Ya que la ideología indígena es ecológica en sus conceptos de integración del humano y de la naturaleza, según ellos no es necesario pedir más para satisfacer el propio placer, ya que el exceso está en contra de la vitalidad de la naturaleza. Así, la sexualidad es una parte natural del placer humano y la ceremonia de la pubertad es el reconocimiento de la entrada a la edad adulta.

Considerando que es necesario fortalecer los principios de justicia y solidaridad, el contrato puede ser utilizado para la protección de las poblaciones vulnerables. La pobreza es actualmente un punto importante de los programas de las agencias internacionales; en el caso del SIDA y del embarazo precoz, es necesario definir el compromiso de estas agencias y de los gobiernos y establecer contratos para la resolución de estos problemas en el marco del respeto de los derechos de estas poblaciones a la sexualidad.

En Panamá, como en muchos países latinoamericanos, la pobreza favorece el hacinamiento familiar en habitaciones de un solo cuarto, y los niños y niñas son testigos tempranos de los rituales de la vida sexual adulta. El incesto es un drama frecuente de la vida de las niñas, reflejo de la negación de su derecho a la autonomía y a la integridad de su cuerpo ${ }^{5}$. Los programas educativos sobre salud sexual y reproductiva (SSR) han incorporado la integridad del cuerpo frente

5 Lastimosamente, no se dispone de cifras oficiales, ya que hay dispersión de las estadísticas entre los diferentes órganos del Estado. a la violencia física, psicológica y sexual, y en la recuperación de la autoestima. En ausencia de violencia personal (haciendo abstracción de la violencia social del hacinamiento), la experiencia de la cotidianidad de la sexualidad adulta favorece una visión de "naturalidad" de la sexualidad y del placer sin privacidad ni límites, lo cual entra en conflicto con los programas de SSR que llaman a una fuerte responsabilidad personal de las acciones. Esta vivencia tampoco prepara a los adolescentes y jóvenes indígenas a enfrentarse a los riesgos de la vida moderna, tales como el SIDA y otras enfermedades de transmisión sexual, cuyas tasas siguen creciendo en estas poblaciones. Por su parte, el embarazo precoz debe considerarse como una remanencia de las sociedades agrícolas con una esperanza de vida baja, que interfiere con las necesidades de las mujeres en la sociedad moderna.

\section{Cultura y placer}

La cultura posmoderna de consumo ofrece una gran variación de formas de placer y celebra a la juventud como una cualidad esencial y necesaria para disfrutar de estos placeres. En este marco, los jóvenes buscan la felicidad en el consumo de alcohol, drogas, velocidad y sexo sin límites $(10,11)$. Cuando fracasan, la sociedad les da la espalda por prejuicios, miedo de su propia imagen o porque el espíritu de competencia eliminó a la solidaridad y la esperanza de una mejor situación. Los adolescentes y jóvenes no entienden esta contradicción y rechazan los programas, que perciben como una "pérdida de tiempo" porque no aportan soluciones perceptibles. Aun cuando asisten por cortesía u obligación, internalizan sólo el contenido que les parece pertinente para su vida. Además, con el desarrollo de la tecnología, los juegos están tan codificados desde la infancia que no permiten el desarrollo de la imaginación propia(12). Quizás sea otro factor para la búsqueda de experiencias imaginativas en las drogas y las conductas peligrosas. 
Las desigualdades de género y el comercio como forma de relación se reflejan en la venta del sexo violento y degradante para las mujeres. Los medios de comunicación juegan un papel importante en esta deshumanización del cuerpo humano y de la sexualidad. Adolescentes y jóvenes adultos están atrapados entre los mensajes de violencia y poder y los discursos morales sobre abstinencia sexual. Ambas posiciones rechazan al placer como un valor y un derecho para el desarrollo humano. La vida acelerada actual no permite el desarrollo de todos los sentidos de una manera equilibrada, guiado por los padres y como preparación a la sexualidad como parte de la integralidad de la vida.

En las conversaciones con dirigentes indígenas, aparecían los conflictos creados por el choque de culturas. Como la mayoría de los hombres panameños, no aceptan que las mujeres decidan por sí mismas sobre su cuerpo y su vida reproductiva, pero, al mismo tiempo, delegan la responsabilidad de la protección de la pareja a las mujeres. Rechazan usar preservativos, por el miedo de que afecte a su masculinidad y a su placer, y son indiferentes a los programas educativos sobre SSR, porque allí asisten principalmente mujeres. El desarrollo de una nueva masculinidad que respete a las mujeres ha sido integrado recientemente en los programas de educación sexual, pero se necesitan más educadores.

Por otra parte, las adolescentes están bajo el modelo de la maternidad diseñado por las iglesias y la tradición, y reforzado por las pocas posibilidades de desarrollo personal. Por lo tanto los programas de prevención de los embarazos en adolescentes han integrado el desarrollo de la autoestima y de la conciencia de la autonomía personal, así como la participación de las mujeres en la vida social. Sin embargo, todavía se percibe un fuerte discurso moral y una negación del placer por parte de los educadores.

\section{La educación sexual}

La visión "represiva" de la educación sexual, que enfatiza en las enfermedades de transmisión sexual, el embarazo precoz y otros peligros de las relaciones sexuales, tiene raíces profundas. Aunque muchos programas actuales han desarrollado una visión "científica", que pone el acento sobre la anatomía y la fisiología de la sexualidad y del embarazo, así como en el desarrollo de la autoestima, parte importante del personal de salud y, más que todo, de los educadores permanece con prejuicios y miedos frente a la sexualidad adolescente. La formación de los educadores sobre el desarrollo psicológico de la niñez hasta la adolescencia es superficial y los psicólogos son escasos en la estructura educativa y de servicios de salud.

Laberge indica que la represión sexual en la educación de los niños se inició en el siglo XVI(14). Para la sociedad de la Edad Media, los niños eran inocentes e indiferentes a la sexualidad hasta la edad de la pubertad, y los adultos podían hablar y conducirse libremente frente a ellos. Esta concepción se encuentra todavía en grupos latinos de bajo nivel socioeconómico y cultural, o en grupos indígenas con menor contacto con la cultura occidental. Los niños empezaron a estudiar la vida sexual conjuntamente con el lenguaje en los "Coloquios" de Erasmus(13). El problema del lenguaje aparece en la confusión, todavía frecuente, entre la prevención de las enfermedades sexuales y el embarazo y la educación sexual o de la sexualidad. Ello puede explicar el rechazo de muchos docentes y padres de familia al desarrollo de estos programas en la escuela: tienen miedo de no poder controlar la sexualidad de los adolescentes y se sienten desestabilizados. Sin embargo, son argumentos que deben ser escuchados para buscar un consenso: ¿capacitación del personal educativo y de los padres, paralelamente a la educación 
de los adolescentes? O ¿desplazamiento de la educación sexual fuera del ámbito escolar?

El concepto moderno de sexualidad en la niñez nació en los siglos XVI y XVII (la inocencia de los niños marchitada por la sociedad), desarrollado por Rousseau y adoptado por la Iglesia católica. A su vez, las iglesias protestantes propusieron el concepto de modestia, castidad y lenguaje correcto. Ambos conceptos perduran todavía en nuestras sociedades latinas. Para Weber, todas estas ideas respondieron al nacimiento del capitalismo moderno, la urbanización de la sociedad moderna y la necesidad de un control de la higiene, a la vez que servían como dominio de la sociedad por parte de los gobiernos(14), dejando los aspectos eróticos de la vida humana al ámbito del teatro, la literatura y el folclor(13).

Las migraciones europeas hacia África y América en esos siglos insistieron en el celibato y la virginidad hasta el matrimonio, para responder a la falta de mujeres y a las largas expediciones de los hombres de la misma categoría étnica, denegando a los indígenas y luego a los afroamericanos el estatuto de ciudadanos de derechos. Si bien estos conceptos han desaparecido de la cultura moderna, los grupos religiosos conservadores han vuelto a insistir sobre la virginidad y la abstinencia, creando conflictos morales entre las obligaciones morales de la fe, la sexualidad en el amor de pareja y las ideas sociales modernas.

Las teorías consecuencialistas y utilitaristas de los siglos XVIII y XIX consideraban que el carácter moral de las acciones humanas está determinado por sus consecuencias y no por alguna característica intrínseca, y que un acto correcto es el que produce el máximo beneficio (felicidad o placer) para el mayor número de personas. El utilitarismo rechaza las prohibiciones morales arbitrarias, basadas sobre prejuicios y no sobre las consecuencias negativas de las acciones ${ }^{6}$.

Actualmente, la teoría utilitarista sirve a los promotores de los derechos de los homosexuales, pero también a grupos conservadores que consideran las consecuencias negativas de la sexualidad de manera aislada. El personal de salud ha olvidado la definición de ésta como "un estado de adaptación a las condiciones del medio ambiente y la resistencia a los peligros de estas condiciones"(15), y el concepto de educación en SSR tiene un aspecto utilitarista: prevenir las enfermedades de transmisión sexual y el embarazo precoz, así como la violencia; por lo tanto, en sus discursos las relaciones sexuales se limitan al coito y otras penetraciones, y a sus peligros. Sólo algunos médicos abordan el concepto de placer. Pero al no aceptar la integración de otros profesionales (sociólogos, antropólogos, psicólogos), no entienden por qué los grupos de adolescentes y jóvenes no responden a sus esfuerzos para la toma de decisiones racionales.

Para Michel Foucault(16), la necesidad de interpretar todos los aspectos de la vida a partir del siglo XVII ha creado "la ciencia de la sexualidad", que analiza todos los aspectos mentales y conductuales de ésta como una forma de represión de la vida sexual y de la autonomía individual. El puritanismo moderno podría ser una forma de rebelión contra la invasión del sexo en todos los aspectos de la vida y la utilización del sexo como objeto comercial. Las empresas publicitarias han asimilado las teorías sobre el subconsciente y el impacto de la repetición y de la asociación de ideas en los mensajes, y, según estas leyes, la sexualidad y el sexo son productos de venta. Pero el respeto al cuerpo humano merece una

6 Luna F, Salles AF. Un mundo complejo. Nuevas reflexiones sobre temas clásicos de bioética. Capítulo 1 (inédito). 
visión integral, más allá que las propuestas de los grupos conservadores. El análisis de los juegos infantiles de Roger Caillois, como "finalidad sin fin" y "placer desinteresado", que no excluye reglas, espacio y tiempo, pero que acepta la incertidumbre, la no producción y la imaginación(17), puede aplicarse para la promoción de la sexualidad responsable con los adolescentes. La responsabilidad individual no sería una carga sino un atributo de la autonomía de decisión y no podría ser utilizada para la represión de la sexualidad.

La bioética puede proponer una educación integral sobre el significado de la vida humana, la sexualidad y el placer, la autonomía y la responsabilidad personal y social, los derechos humanos y los derechos sexuales y reproducti- vos, así como sobre la integridad de la niñez. La ética del placer significa ética de la imaginación y del juego, de la sensualidad y de la sexualidad, de la ciencia y de los derechos humanos, como parte de un proyecto de sociedad centrado sobre el ser humano. Implica la autonomía y la justicia, ya que puede aplicarse a cada individuo sin significar egoísmo, porque necesita del diálogo con los demás. Los programas de educación sobre salud sexual y reproductiva deben enmarcarse en los derechos humanos, los derechos de la niñez y la equidad de género. En su aplicación concreta deben evitar las acciones autoritarias, unilaterales y las contradicciones entre conceptos negativos frente a la vivencia de placer de los adolescentes y jóvenes, y buscar un consenso que no sacrifique sus derechos a los intereses de los grupos conservadores.

\section{Referencias}

1. Dirección de Estadísticas y Censo. Censo nacional, 2000. Panamá: Contraloría de la Nación; 2001.

2. UNFPA. Fast Facts on Maternal Mortality and Morbidity, 2003, En: UNFPA. State of the World Population; 2003.

3. WHO Facts Sheet. Young People and Sexually Transmitted Diseases. WHO; 1997.

4. United Nations. Report of the International Conference on Population and Development, Cairo 5-13 September 1994. United Nations publication; 1994.

5. United Nations. Programme of Action of the International Conference on Population and Development, Cairo, Egypt, Sept. 5-13, 1994. Part Two: Statements and Reservations on the Programme of Action, Holy See, Libyan Arab Jamahiriya and Yemen, U.N. Doc. A/CONF.171/13; 1994.

6. IWHC. Beijing Plus Five: IWHC's Analysis of Negotiations and Final "Further Actions". Document $5 ; 2000$.

7. The Swedish Association for Sexuality Education (RFSU). Breaking Through. A Guide to Sexual and Reproductive Health and Rights. Stockholm: Ylva Bergman; 2004.

8. Singh JS. Creating a new consensus on population. The International Conference on Population. and Development. London: Earthscan; 1998.

9. UNFPA. Facts Sheet: Meeting the Demand for Reproductive Health Essentials. 2003.

10. Winer RL, et al. Genital human papillomavirus infection: incidence and risk factors in a cohort of female university students. American Journal of Epidemiology 2003: 157(3): 218-226. 
11. Hingson R, et al. Early age of first drunkenness as a factor in college students' unplanned and unprotected sex attributable to drinking. Pediatrics 2003; 111(1): 34-41.

12. Proulx J. Le jeu, le rite, la fête. Critère 1971; 3.

13. Laberge H. La sexualité à travers les âges. En: Dufresne J, Dumont F, Martin Y. Traité d'anthropologie médicale. Québec: Institut québécois de recherche sur la culture, Presses de l'Université du Québec; 1985 : 709-732.

14. Weber M. L'Éthique protestante et l'esprit du capitalisme. Paris: Plon ; 1964.

15. Dubos R. Définitions de la santé. Critère 1976; 15.

16. Foucault M. La volonté de savoir. Histoire de la sexualité. Paris: Gallimard; 1976: 24.

17. Caillois R. Les Jeux et les hommes. Paris: Gallimard Edition; 1958: 31-45.

Recibido el 13 de agosto de 2006.

Aceptado el 12 de marzo de 2007. 